\title{
Structure of potamoplankton along a gradient of preservation of riparian vegetation in subtropical streams
}

\author{
DENISE PERESIN ${ }^{1}$, LEZILDA C. TORGAN ${ }^{2}$, UWE H. SCHULZ ${ }^{1}$ and LUCIANE O. CROSSETTI \\ ${ }^{1}$ Programa de Pós-Graduação em Biologia, Universidade do Vale do Rio dos Sinos, \\ Av. Unisinos, 950, Cristo Rei, 93022-000 São Leopoldo, RS, Brasil \\ ${ }^{2}$ Fundação Zoobotânica do Rio Grande do Sul, Rua Dr. Salvador França, 1427, Caixa Postal 1188, \\ Jardim Botânico, 90690-000 Porto Alegre, RS, Brasil \\ ${ }^{3}$ Departamento de Ecologia, Universidade Federal do Rio Grande do Sul, Caixa Postal 15007, \\ Agronomia, 91501-970 Porto Alegre, RS, Brasil
}

Manuscript received on August 19, 2013; accepted for publication on January 3, 2014

\begin{abstract}
Water quality in lotic and lentic systems is greatly influenced by the land use in the adjacent areas. This study was conducted in order to assess whether different degrees of preservation of riparian forest (from well preserved with $30 \mathrm{~m}$ wide up to degraded with $<5 \mathrm{~m}$ wide) influence potamoplankton community structure. The study was conducted in three streams in the upper region of the Rio dos Sinos basin, at four sampling sites, with different width of the forest. Samplings were gathered for abiotic and biological analysis in winter (August 2010) and summer (January/February 2011). The analysis of environmental and biotic variables indicated that seasonality was the main determinant of biomass patterns, regardless of the width of riparian vegetation. The potamoplankton community of the studied streams responded to environmental conditions, although lack of significant difference among the different degrees of riparian vegetation preservation has been observed. Increasing trends on biomass, richness and diversity of species, and higher concentrations of total phosphorus and nitrogen in the non-preserved areas might indicate that the different gradients of preservation of riparian vegetation are related with the water quality in subtropical streams and consequently with the potamoplankton structure.
\end{abstract}

Key words: riparian forest, water quality, stream, bioindicator.

\section{INTRODUCTION}

The degradation of riparian vegetation causes serious impacts to aquatic ecosystems worldwide. Areas of riparian forests are important for the retention of nutrients and sediments (Lowrance et al. 1997, Storey and Cowley 1997, Sabater et al. 2003, Naiman et al. 2005, Dosskey et al. 2011). The efficiency of these areas in the control of nitrogen

Correspondence to: Luciane Oliveira Crossetti

E-mail: luciane.crossetti@ufrgs.br
(N) and phosphorous (P), as well as chemical contaminants, is strongly influenced by its width (Borin et al. 2004, Gorsevski et al. 2008, Newbold et al. 2010). Alterations of the structure and dynamics of aquatic communities are the consequences.

Several studies have reported on the influence of riparian vegetation on the water quality and on the response of the phytoplankton community (Naiman et al. 2005, Dudgeon 2008). The influence of different land uses 
on the community of diatoms in tropical and temperate rivers (Vázquez et al. 2011, Gabel et al. 2012) has been observed, and an increase in the proportion of $\mathrm{N}$ and $\mathrm{P}$ when compared to silicate $(\mathrm{Si})$, may result in a decline of diatoms (Conley and Malone 1992, Cugier et al 2005). Moreover, changes in the nutrient loadings caused by deforestation of up to $50 \%$ of riparian forests may enhance eutrophication and probably increase the frequency of algal blooms (Perry et al. 1999).

Historically, the phytoplankton from lotic ecosystems have received less attention in limnological studies (Rodrigues et al. 2007). In Brazil, most of them refer to the taxonomical (Bittencourt-Oliveira and Castro 1993, BittencourtOliveira 1993a, b, Mendes-Câmara et al. 2002, Soares et al. 2007) and to the ecological approaches, focusing on diversity (Bittencourt-Oliveira 2002, Borges et al. 2003), structure and the dynamics of phytoplankton (Train and Rodrigues 1998, Silva et al. 2001, Soares et al. 2007). Nevertheless, studies addressing the relations between the preservation of the riparian vegetation and its consequences on the potamoplankton structure are incipient in Brazilian limnology.

In this context, the aim of this study was to evaluate the structure of potamoplankton along a gradient of preservation of riparian vegetation in subtropical streams. Hypothetically, in the stream stretches with preserved riparian vegetation, lower potamoplankton biomass is expected, as well as lower richness and species diversity owing to the lower concentration of nutrients and water transparency. These conditions are possible due to the ability of riparian vegetation of retaining nutrients and sediments, and to provide shading. Unlike, areas with little or no riparian vegetation might present greater potamoplankton biomass, richness and diversity of species, due to the higher concentration of nutrients, turbidity and lightness in the water.

\section{MATERIALS AND METHODS}

STUdied AREA, DELIMITATION AND SAMPLING FREQUENCY

Samples were collected in three streams in the upper reaches of the Sinos River basin, located in the eastern portion of the State of Rio Grande do Sul. In the region higher altitudes are observed, human occupation is less intense and the land use is extensive agriculture.

The evaluated streams were: (i) Padilha stream (29³2'11.94 "S, 50³9'50.07" W), located in the municipality of Taquara, (ii) Areias stream $\left(29^{\circ} 35^{\prime} 09.56\right.$ "S, 50³3'32.59 "W), located in the municipality of Rolante, (iii) Chuvisqueiro stream $\left(29^{\circ} 36^{\prime} 11.66^{\prime \prime} \mathrm{S}, 50^{\circ} 28^{\prime} 55.89\right.$ "W), located between the towns of Riozinho and Rolante (Figure 1). In each of the streams, samples were performed at four sites with different widths of riparian vegetation. The sites were numbered in upstream - downstream direction based on the following gradient: site 1 (P1) well preserved riparian vegetation, more than $30 \mathrm{~m}$ wide; site 2 (P2) riparian vegetation partially conserved, width between 15 and $30 \mathrm{~m}$; site 3 (P3) riparian vegetation poorly conserved, width between 5 and $15 \mathrm{~m}$; site 4 (P4) degraded riparian vegetation, width $<5 \mathrm{~m}$. The land uses in the adjacent areas of the sample sites were predominantly extensive agriculture and pastures. The distance between the sample sites was of at least $1 \mathrm{~km}$.

According to the Strahler method (1957), the sampled points were classified as stream orders 3 and order 4. Samples were collected in winter (August 2010) and summer (January / February 2011).

\section{ABIOTIC ANALYSIS}

In situ, the following set of variables were measured with a multi water quality probe (Horiba U53): $\mathrm{pH}$, dissolved oxygen (DO), water temperature (Temp $\mathrm{H}_{2} \mathrm{O}$ ), conductivity (Cond), redox potential (ORP), total dissolved solids (TDS) and turbidity (Turb). Water velocity (Vel. $\mathrm{H}_{2} \mathrm{O}$ ) was measured 


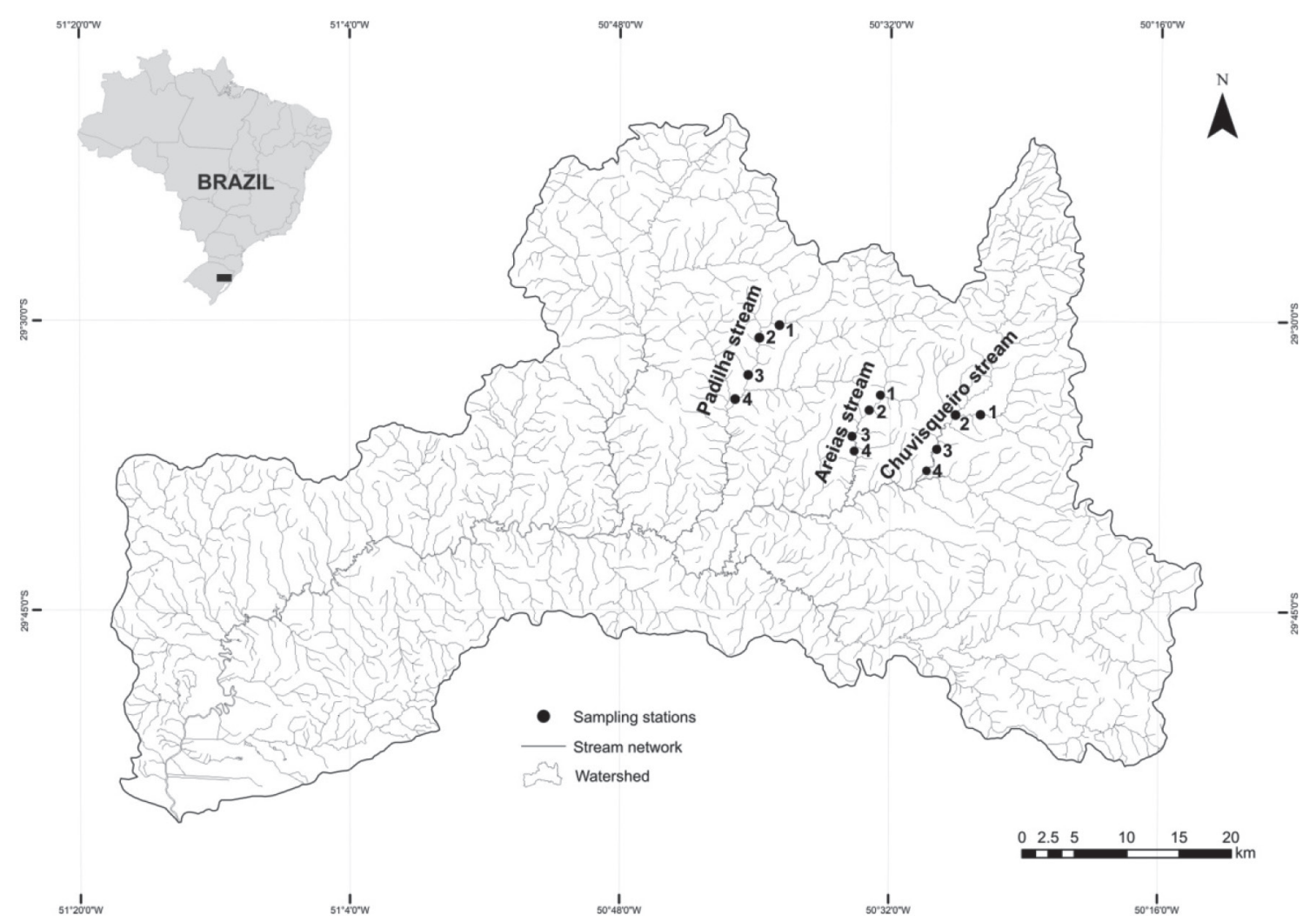

Figure 1 - Sinos River watershed delimitation and sampled streams.

with an electronic flow meter (Flo-Mate 2000, Marsh_McBirney) equipment. The stream width was also registered at each sampled point. Water samples were collected for analysis, in laboratory, of orthophosphate (SRP), total phosphorus $(\mathrm{TP})$, total nitrogen $(\mathrm{TN})$, organic nitrogen $(\mathrm{ON})$, ammonia $\left(\mathrm{N}-\mathrm{NH}_{3}{ }^{-}\right)$, nitrite $\left(\mathrm{N}-\mathrm{NO}_{2}{ }^{-}\right)$(APHA 2005) and nitrate $\left({\mathrm{N}-\mathrm{NO}_{3}}^{-}\right)$(Method of salicylate). In the summer period, analyses of organic phosphorus (OP), chlorophyll a (Cloa) and silica (Si) (APHA 2005) were included. The total phosphorus and nitrogen load, considering the flow (Bain and Stevenson, 1999), was estimated in the first week of July 2012. The load was calculated by the "average sample load" model ( $\mathrm{Li}$ et al. 2003): $\mathrm{L}=\mathrm{K}(\mathrm{C} * \mathrm{Q})$, where $\mathrm{L}=$ load, $\mathrm{K}=$ time interval (in this case, one day), $\mathrm{C}=$ concentration and $\mathrm{Q}=$ discharge.
The average temperature and precipitation data for the months of August 2010 and February 2011 were obtained from Fundação Estadual de Pesquisa Agropecuária - FEPAGRO (FEPAGRO 2010, 2011). The data correspond to the station located in Campo Bom, which is the nearest to the sampling sites.

BiOLOGICAL ANALYSIS

The samples used to determine the phytoplankton floristic composition, were collected with a plankton net and fixed with aqueous formalin 3-5\%. The identification of diatoms was performed after the clarification of the collected material as described by the Müller-Melches and Ferrando (1956) technique, without the addition of sulfuric acid. The permanent slides were mounted using Naphrax ${ }^{\circledR}$. Diatoms were identified using specialized literature. 
Samples for quantitative analysis of phytoplankton were collected in the subsurface water $( \pm 10 \mathrm{~cm}$ surface). The samples were fixed with acetic Lugol's solution 1\%. Phytoplankton quantification followed Utermöhl (1958) and sedimentation time was set according to Lund et al. (1958), who defined 4 hours for each centimeter of chamber height. The organisms density (individuals.mL-1 ${ }^{1}$ ) was calculated according to Ros (1979). The biovolume ( $\mathrm{mm}^{3}$. $\mathrm{mL}^{-1}$ ) of each species was calculated based on the geometry form that best approximated to the cell shape, isolated or combined, according to Hillebrand et al. (1999). Descriptive species were considered those that contributed most to the total biomass $(>1 \%)$ in each sampled point. The richness (number of taxa) and diversity index (H') (Shannon and Weaver 1963) were also calculated using Past - statistical software (Hammer et al. 2001).

\section{DATA ANALYSIS}

The data were analyzed through descriptive statistics. In order to compare the biological and abiotic parameters from different sampled points, the Analysis of Variance (ANOVA "one way") was performed, using the statistical software SYSTAT 12 for Windows, version 12 (SYSTAT Software 2007). The streams were treated as replicas $(\mathrm{n}=3)$. A Detrended Correspondence Analysis (DCA) was performed to indicate the need to use a unimodal method or linear ordering. From its results (length of gradient: 5.0), the Canonical Correspondence Analysis (CCA) was performed to verify the relationships between descriptive species and key environmental variables in the studied areas. Therefore, the biological and abiotic variables were transformed (ranging and $\log \mathrm{x}+1$, respectively), avoiding colinearity. The PC-ORD statistical software, version 5.15 (McCune and Mefford 2006) was used to perform these analyses.

\section{RESULTS}

ABIOTIC VARIABLES

Considering the variation of the physic-chemical data in different gradients of riparian forest preservation, an increasing trend in water temperature, conductivity, turbidity and TSD (Table I) was observed, from P1 (most preserved) to P4 (less preserved). On the other hand, DO concentrations tended to decrease from $\mathrm{P} 1$ to $\mathrm{P} 4$, although not significantly $(\mathrm{p}=0.215$ in winter and $\mathrm{p}=0.243$ in summer). Water temperature and $\mathrm{N}-\mathrm{NO}_{2}{ }^{-}$had higher values in summer, while the DO and TP had higher concentrations in winter (Table I). There was an increase in the TP concentrations, from three to five times in winter, when compared to summer. Water velocity showed a significant difference between the sampled points in winter $(\mathrm{F}=10.780, \mathrm{p}=0.030)$, being higher in the most preserved point (P1). The values of total nitrogen and total phosphorus loads were significantly higher in points less preserved ( $\mathrm{F}$ $=4.56, \mathrm{p}=0.038 ; \mathrm{F}=5.33, \mathrm{p}=0.026$ ), being $\mathrm{P} 1$ and $\mathrm{P} 4$ statistically different for both nutrients $(\mathrm{p}=0.036$ and 0.020 , respectively) (Figure 2A).

\section{BIOLOGICAL RESPONSES}

A total of 151 taxa were identified, distributed in 10 taxonomic classes. Bacillariophyceae had the highest taxa number (29\% of all identified taxa), followed by Chlorophyceae (26\%), Euglenophyceae $(12 \%)$ and Cyanobacteria (10\%).

The potamoplankton biomass was higher at all sites in summer (Table I, Figure 2B) and the highest mean value $\left(0.08 \mathrm{~mm}^{3} \mathrm{~L}^{-1}\right)$ was found at $\mathrm{P} 3$. However, regardless of the season, there were no significant differences in biomass in relation to the gradient of riparian forest preservation $(p=0.793$ in winter and $p=0.713$ in summer). The same trend was registered for the values of chlorophyll- $a$ that had lower average values in $\mathrm{P} 1\left(0.50 \mu \mathrm{gL}^{-1}\right)$ compared to P3 $\left(1.0 \mu \mathrm{gL}^{-1}\right)$ and $\mathrm{P} 4\left(0,7 \mu \mathrm{gL}^{-1}\right)$, although not statistically significant ( $\mathrm{p}=0.262$ in summer). 
TABLE I

Minimum, maximum, average and standard deviation of abiotic variables for each sampling (season) in each stream $(n=3)$.

\begin{tabular}{|c|c|c|c|c|c|c|c|c|}
\hline \multirow{2}{*}{$\begin{array}{l}\text { Season } \\
\text { Areas }\end{array}$} & \multicolumn{4}{|c|}{ Winter } & \multicolumn{4}{|c|}{ Summer } \\
\hline & 1 & 2 & 3 & 4 & 1 & 2 & 3 & 4 \\
\hline $\begin{array}{l}\text { Total } \\
\text { precipitation } \\
\left(\mathrm{mm} \cdot \mathrm{month}^{-1}\right)\end{array}$ & \multicolumn{4}{|c|}{53.6} & \multicolumn{4}{|c|}{154.4} \\
\hline $\begin{array}{l}\text { Average air } \\
\text { temperature } \\
\left({ }^{\circ} \mathrm{C} . \mathrm{month}^{-1}\right)\end{array}$ & \multicolumn{4}{|c|}{16.3} & \multicolumn{4}{|c|}{26.8} \\
\hline $\begin{array}{l}\text { Water } \\
\text { temperature } \\
\left({ }^{\circ} \mathrm{C}\right)\end{array}$ & $\begin{array}{l}10.0-12.2 \\
(11.2 ; \pm 1.2)\end{array}$ & $\begin{array}{l}11.0-12.6 \\
(11.9 ; \pm 0.8)\end{array}$ & $\begin{array}{l}11.4-12.8 \\
(12.0 ; \pm 0.7)\end{array}$ & $\begin{array}{l}12.0-13.3 \\
(12.7 ; \pm 0.7)\end{array}$ & $\begin{array}{l}21.6-24.2 \\
(22.8 ; \pm 1.3)\end{array}$ & $\begin{array}{l}22.8-24.4 \\
(23.8 ; \pm 0.9)\end{array}$ & $\begin{array}{l}22.2-24.5 \\
(23.6 ; \pm 1.3)\end{array}$ & $\begin{array}{l}22.9-24.6 \\
(23.8 ; \pm 0.9)\end{array}$ \\
\hline $\mathrm{DO}\left(\mathrm{mg} \cdot \mathrm{L}^{-1}\right)$ & $\begin{array}{l}12.7-15.1 \\
(14.1 ; \pm 1.2)\end{array}$ & $\begin{array}{l}12.2-14.7 \\
(13.7 ; \pm 1.3)\end{array}$ & $\begin{array}{l}12.1-15.4 \\
(13.8 ; \pm 1.6)\end{array}$ & $\begin{array}{l}12.0-13.5 \\
(12.8 ; \pm 0.8)\end{array}$ & \begin{tabular}{|l}
$7.7-9.3$ \\
$(8.7 ; \pm 0.9)$
\end{tabular} & $\begin{array}{l}7.3-8.1 \\
(7.7 ; \pm 0.4)\end{array}$ & $\begin{array}{l}7.5-7.6 \\
(7.5 ; \pm 0.0)\end{array}$ & $\begin{array}{l}7.2-8.6 \\
(7.8 ; \pm 0.7)\end{array}$ \\
\hline $\begin{array}{l}\text { Conductivity } \\
\left(\mathrm{ms} \cdot \mathrm{cm}^{-1}\right)\end{array}$ & $\begin{array}{l}0.036-0.046 \\
(0.040 ; \pm \\
0.006)\end{array}$ & $\begin{array}{l}0.037-0.052 \\
(0.043 ; \pm \\
0.008)\end{array}$ & $\begin{array}{l}0.039-0.057 \\
(0.045 ; \pm \\
0.010)\end{array}$ & $\begin{array}{l}0.044-0.061 \\
(0.053 ; \pm \\
0.009)\end{array}$ & $\begin{array}{l}0.040-0.048 \\
(0.044 ; \pm \\
0.004)\end{array}$ & $\begin{array}{l}0.040-0.053 \\
(0.047 ; \pm \\
0.007)\end{array}$ & $\begin{array}{l}0.044-0.057 \\
(0.049 ; \\
\pm 0.007)\end{array}$ & $\begin{array}{l}0.045-0.065 \\
(0.053 ; \pm \\
0.011)\end{array}$ \\
\hline TDS $\left(\mathrm{g} \cdot \mathrm{L}^{-1}\right)$ & $\begin{array}{l}0.023-0.030 \\
(0.026 ; \pm \\
0.004)\end{array}$ & $\begin{array}{l}0.024-0.034 \\
(0.028 ; \pm \\
0.006)\end{array}$ & $\begin{array}{l}0.025-0.037 \\
(0.029 ; \pm \\
0.007)\end{array}$ & $\begin{array}{l}0.029-0.040 \\
(0.035 ; \pm \\
0.006)\end{array}$ & $\begin{array}{l}0.026-0.031 \\
(0.028 ; \pm \\
0.003)\end{array}$ & $\begin{array}{l}0.026-0.035 \\
(0.031 ; \pm \\
0.005)\end{array}$ & $\begin{array}{l}0.029-0.037 \\
(0.032 ; \pm \\
0.005)\end{array}$ & $\begin{array}{l}0.030-0.042 \\
(0.034 ; \pm \\
0.007)\end{array}$ \\
\hline $\mathrm{pH}$ & $\begin{array}{l}7.1-8.4 \\
(7.5 ; \pm 0.7)\end{array}$ & $\begin{array}{l}7.2-7.5 \\
(7.3 ; \pm 0.2)\end{array}$ & $\begin{array}{l}7.2-7.6 \\
(7.4 ; \pm 0.2)\end{array}$ & $\begin{array}{l}7.2-7.5 \\
(7.4 ; \pm 0.2)\end{array}$ & $\begin{array}{l}7.1-7.2 \\
(7.2 ; \pm 0.1)\end{array}$ & $\begin{array}{l}7.0-7.6 \\
(7.3 ; \pm 0.3)\end{array}$ & $\begin{array}{l}7.0-7.7 \\
(7.3 ; \pm 0.3)\end{array}$ & $\begin{array}{l}7.1-7.6 \\
(7.3 ; \pm 0.3)\end{array}$ \\
\hline $\begin{array}{l}\text { Turbidity } \\
\text { (NTU) }\end{array}$ & $\begin{array}{l}2.4-10.1 \\
(6.6 ; \pm 3.9) \\
\end{array}$ & $\begin{array}{l}3.0-8.0 \\
(4.7 ; \pm 2.8)\end{array}$ & $\begin{array}{l}3.0-8.0 \\
(4.7 ; \pm 2.8)\end{array}$ & $\begin{array}{l}4.0-11.3 \\
(7.7 ; \pm 3.7)\end{array}$ & $\begin{array}{l}0.3-12.2 \\
(5.9 ; \pm 6.0)\end{array}$ & $\begin{array}{l}1.3-12.4 \\
(6.1 ; \pm 5.7)\end{array}$ & $\begin{array}{l}0.4-14.5 \\
(6.3 ; \pm 7.3)\end{array}$ & $\begin{array}{l}0.5-14.3 \\
(6.3 ; \pm 7.2)\end{array}$ \\
\hline $\begin{array}{l}\text { Stream } \\
\text { width (m) }\end{array}$ & $\begin{array}{l}0.26-0.38 \\
(0.32 ; \pm 0.06)\end{array}$ & $\begin{array}{l}0.30-0.40 \\
(0.34 ; \pm 0.05)\end{array}$ & $\begin{array}{l}0.16-0.22 \\
(0.19 ; \pm 0.03)\end{array}$ & $\begin{array}{l}0.17-0.22 \\
(0.20 ; \pm 0.03)\end{array}$ & \begin{tabular}{|l|}
$0.22-0.35$ \\
$(0.31 ; \pm 0.08)$
\end{tabular} & $\begin{array}{l}0.18-0.41 \\
(0.26 ; \pm 0.13)\end{array}$ & $\begin{array}{l}0.07-0.42 \\
(0.28 ; \pm 0.18)\end{array}$ & $\begin{array}{l}0.26-0.38 \\
(0.30 ; \pm 0.07)\end{array}$ \\
\hline $\begin{array}{l}\text { SRP } \\
\left(\mu g . L^{-1}\right)\end{array}$ & $\begin{array}{l}9-80 \\
(39 ; \pm 37)\end{array}$ & $\begin{array}{l}3-80 \\
(34 ; \pm 40)\end{array}$ & $\begin{array}{l}4-40 \\
(25 ; \pm 19)\end{array}$ & $\begin{array}{l}12-50 \\
(31 ; \pm 19)\end{array}$ & $\begin{array}{l}20-60 \\
(37 ; \pm 21)\end{array}$ & $\begin{array}{l}30-30 \\
(30 ; \pm 0)\end{array}$ & $\begin{array}{l}30-60 \\
(43 ; \pm 15)\end{array}$ & $\begin{array}{l}20-40 \\
(30 ; \pm 10)\end{array}$ \\
\hline $\begin{array}{l}\text { TP } \\
\left(\mu \mathrm{g} . \mathrm{L}^{-1}\right)\end{array}$ & $\begin{array}{l}110-190 \\
(147 ; \pm 40)\end{array}$ & $\begin{array}{l}100-180 \\
(140 ; \pm 40)\end{array}$ & $\begin{array}{l}130-200 \\
(173 ; \pm 38)\end{array}$ & $\begin{array}{l}110-190 \\
(140 ; \pm 44)\end{array}$ & $\begin{array}{l}27-37 \\
(33 ; \pm 6)\end{array}$ & $\begin{array}{l}23-38 \\
(31 ; \pm 8)\end{array}$ & $\begin{array}{l}34-66 \\
(45 ; \pm 18)\end{array}$ & $\begin{array}{l}23-33 \\
(27 ; \pm 5)\end{array}$ \\
\hline $\begin{array}{l}\mathrm{N}-\mathrm{NO}_{3}^{-} \\
\left(\mu \mathrm{g} \cdot \mathrm{L}^{-1}\right)\end{array}$ & $\begin{array}{l}450-560 \\
(503 ; \pm 55)\end{array}$ & $\begin{array}{l}400-750 \\
(527 ; \pm 194)\end{array}$ & $\begin{array}{l}350-820 \\
(513 ; \pm 266)\end{array}$ & $\begin{array}{l}370-520 \\
(420 ; \pm 87)\end{array}$ & \begin{tabular}{|l}
$200-540$ \\
$(326.7 ; \pm$ \\
$185.8)$
\end{tabular} & $\begin{array}{l}200-560 \\
(320.0 ; \pm \\
207.8)\end{array}$ & $\begin{array}{l}200-900 \\
(443.3 ; \pm \\
395.8)\end{array}$ & $\begin{array}{l}200-220 \\
(206.7 ; \pm \\
11.5)\end{array}$ \\
\hline $\begin{array}{l}\text { N-NO }{ }_{2}^{-} \\
\text {(ug. } \mathrm{L}^{-1} \text { ) }\end{array}$ & $\begin{array}{l}1.2-2.3 \\
(1.7 ; \pm 0.6)\end{array}$ & $\begin{array}{l}0.3-3.4 \\
(2.0 ; \pm 1.6)\end{array}$ & $\begin{array}{l}0.1-2.5 \\
(1.2 ; \pm 1.2)\end{array}$ & $\begin{array}{l}2.2-3.4 \\
(2.7 ; \pm 0.6)\end{array}$ & $\begin{array}{l}10-13 \\
(11 ; \pm 2)\end{array}$ & $\begin{array}{l}10-12 \\
(11 ; \pm 1)\end{array}$ & $\begin{array}{l}10-10 \\
(10 ; \pm 0)\end{array}$ & $\begin{array}{l}10-14 \\
(11 ; \pm 2)\end{array}$ \\
\hline $\begin{array}{l}\mathrm{N}^{-\mathrm{NH}_{3}}{ }^{-} \\
\left(\mu \mathrm{g} . \mathrm{L}^{-1}\right)\end{array}$ & $\begin{array}{l}90-110 \\
(103 ; \pm 12)\end{array}$ & $\begin{array}{l}80-140 \\
(107 ; \pm 31)\end{array}$ & $\begin{array}{l}80-220 \\
(147 ; \pm 70)\end{array}$ & $\begin{array}{l}70-150 \\
(120 ; \pm 44)\end{array}$ & $\begin{array}{l}230-420 \\
(320 ; \pm 95)\end{array}$ & $\begin{array}{l}300-1.420 \\
(763 ; \pm 584)\end{array}$ & $\begin{array}{l}420-1.460 \\
(793 ; \pm 579)\end{array}$ & $\begin{array}{l}190-1.570 \\
(740 ; \pm 731)\end{array}$ \\
\hline $\mathrm{TN}\left(\mu \mathrm{g} \cdot \mathrm{L}^{-1}\right)$ & $\begin{array}{l}380-450 \\
(403 ; \pm 40)\end{array}$ & $\begin{array}{l}120-650 \\
(443 ; \pm 284)\end{array}$ & $\begin{array}{l}430-660 \\
(563 ; \pm 119)\end{array}$ & $\begin{array}{l}140-770 \\
(547 ; \pm 353)\end{array}$ & $\begin{array}{l}610-2,210 \\
(1,170 ; \pm 902)\end{array}$ & $\begin{array}{l}690-4,260 \\
(1,897 ; \pm \\
2,047)\end{array}$ & $\begin{array}{l}780-1.610 \\
(1,067 ; \pm 471)\end{array}$ & $\begin{array}{l}600-2.340 \\
(1,213 ; \pm 977)\end{array}$ \\
\hline $\mathrm{DIN}\left(\mu \mathrm{g} . \mathrm{L}^{-1}\right)$ & $\begin{array}{l}562-671 \\
(608 ; \pm 56) \\
\end{array}$ & $\begin{array}{l}500-833 \\
(635 ; \pm 175)\end{array}$ & $\begin{array}{l}430-1,041 \\
(661 ; \pm 332)\end{array}$ & $\begin{array}{l}512-593 \\
(543 ; \pm 44)\end{array}$ & $\begin{array}{l}440-863 \\
(658 ; \pm 212)\end{array}$ & $\begin{array}{l}780-1,992 \\
(1,094 ; 789)\end{array}$ & $\begin{array}{l}660-2,370 \\
(1,247 ; \pm 973)\end{array}$ & $\begin{array}{l}400-1,804 \\
(958 ; \pm 745)\end{array}$ \\
\hline $\mathrm{Si}\left(\mathrm{mg} \cdot \mathrm{L}^{-1}\right)$ & - & - & - & - & $\begin{array}{l}4.83-7.24 \\
(6.2 ; \pm 1.2)\end{array}$ & $\begin{array}{l}6.43-8.29 \\
(7.2 ; \pm 1.0)\end{array}$ & $\begin{array}{l}6.71-9.32 \\
(7.8 ; \pm 1.4)\end{array}$ & $\begin{array}{l}6.80-8.57 \\
(7.5 ; \pm 0.9)\end{array}$ \\
\hline $\begin{array}{l}\text { Biomass } \\
\left(\mathrm{mm}^{3} \cdot \mathrm{L}^{-1}\right)\end{array}$ & $\begin{array}{l}0.001-0.038 \\
(0.015 ; \pm \\
0.020)\end{array}$ & $\begin{array}{l}0.001-0.011 \\
(0.006 ; \pm \\
0.005)\end{array}$ & $\begin{array}{l}0.003-0.022 \\
(0.011 ; \pm \\
0.010)\end{array}$ & $\begin{array}{l}0.001-0.017 \\
(0.009 ; \pm \\
0.008)\end{array}$ & $\begin{array}{l}0.026-0.063 \\
(0.039 ; \pm \\
0.021)\end{array}$ & $\begin{array}{l}0.038-0.105 \\
(0.060 ; \pm \\
0.038)\end{array}$ & $\begin{array}{l}0.030-0.117 \\
(0.079 ; \pm \\
0.084)\end{array}$ & $\begin{array}{l}0.036-0.047 \\
(0.041 ; \pm \\
0.006)\end{array}$ \\
\hline Chlorophyll- $a$ & - & - & - & - & $\begin{array}{l}0.50-0.50 \\
(0.5 ; \pm 0.0)\end{array}$ & $\begin{array}{l}0.50-1.03 \\
(0.8 ; \pm 0.3)\end{array}$ & $\begin{array}{l}0.59-1.32 \\
(1.0 ; \pm 0.4)\end{array}$ & $\begin{array}{l}0.50-1.13 \\
(0.7 ; \pm 0.4)\end{array}$ \\
\hline $\begin{array}{l}\text { Water } \\
\text { velocity }\end{array}$ & $\begin{array}{l}0.22-0.35 \\
(0.31 ; \pm 0.06)\end{array}$ & $\begin{array}{l}0.18-0.41 \\
(0.26 ; \pm 0.11)\end{array}$ & $\begin{array}{l}0.07-0.42 \\
(0.28 ; \pm 0.15)\end{array}$ & $\begin{array}{l}0.26-0.38 \\
(0.30 ; \pm 0.06)\end{array}$ & $\begin{array}{l}0.26-0.38 \\
(0.32 ; \pm 0.05)\end{array}$ & $\begin{array}{l}0.30-0.40 \\
(0.34 ; \pm 0.04)\end{array}$ & $\begin{array}{l}0.16-0.22 \\
(0.19 ; \pm 0.02)\end{array}$ & $\begin{array}{l}0.17-0.22 \\
(0.20 ; \pm 0.02)\end{array}$ \\
\hline
\end{tabular}


A
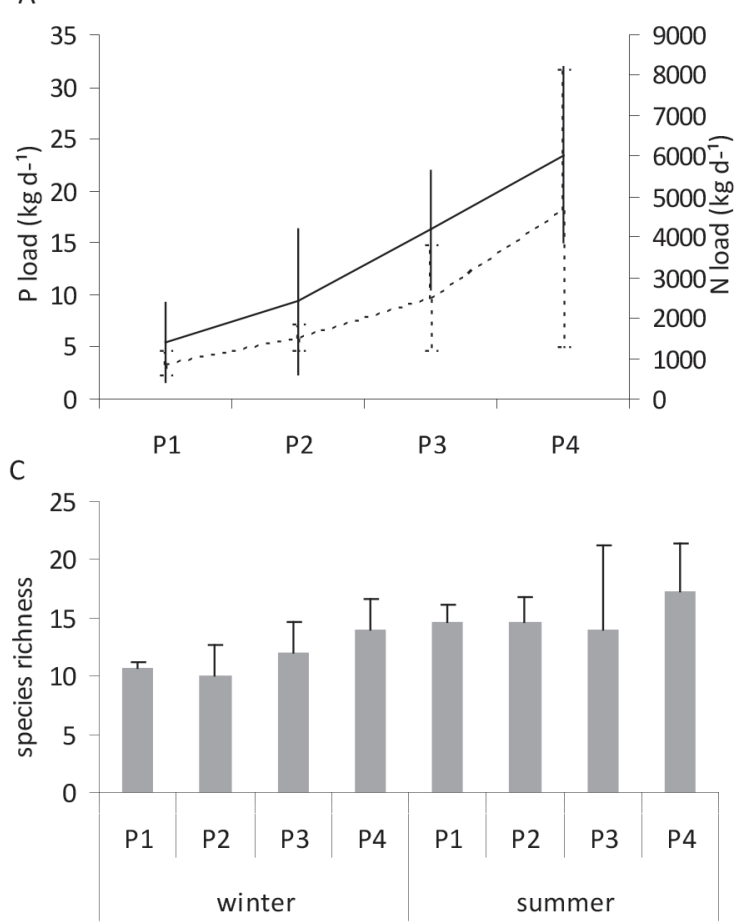

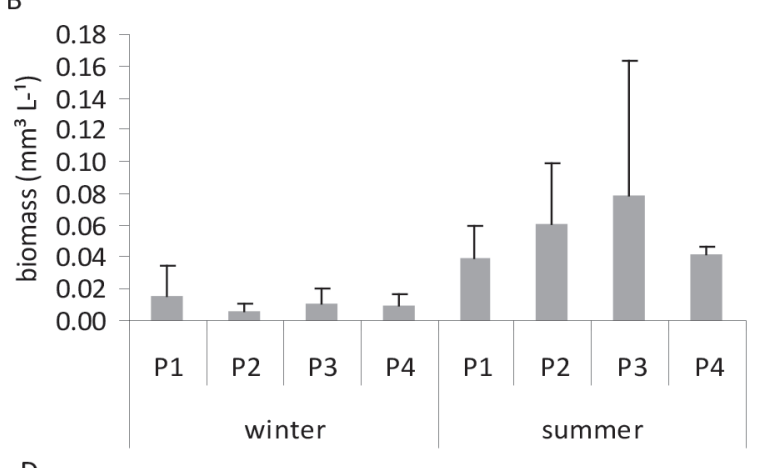

D

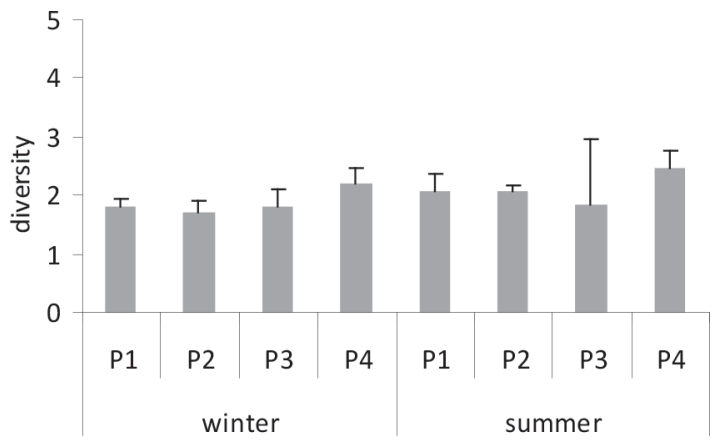

Figure 2 - Phosphorus (P load, kg.d $\left.\mathrm{d}^{-1}\right)$ and nitrogen loads $\left(\mathrm{N}\right.$ load,kg.d $\left.\mathrm{d}^{-1}\right)(\mathrm{A})$, biomass $\left(\mathrm{mm}^{3} \cdot \mathrm{L}^{-1}\right)(\mathrm{B})$, species richness (number of individuals) $(\mathrm{C})$ and diversity $\left(\mathrm{mg}\right.$ ind $\left.^{-1}\right)(\mathrm{D})$ estimated for each sampled point $(\mathrm{P} 1=$ most preserved; P4 $=$ less preserved).

The richness and diversity (Figures $2 \mathrm{C}$ and 2D) varied depending on the gradient of riparian forest preservation, regardless of the season of the year, although not significantly $(\mathrm{p}>0.05)$. P4 showed the highest richness and diversity of species in winter (14 species and 2.2 bits $\mathrm{mg}^{-1}$, respectively) and in

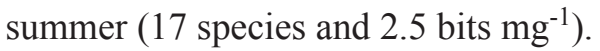

A total of 24 descriptive species were identified (Table II). No relationship was observed between the biomass of descriptive species and the gradient of riparian forest preservation. Comparing the two seasons analyzed (Table II), a replacement of the most descriptive species was observed in each one of the analyzed sites. Some species as Cocconeis placentula var. acuta F. Meister and Ulnaria ulna (Nitzs.) Comp. occurred in both summer and winter, while the species Amphipleura lindheimeri Grun., Gomphonema parvulum Kütz. and Closterium moniliferum (Ehr.) Ralfs showed preference for summer.

\section{INTEGRATED ANALYSIS}

The CCA was performed using the seven major environmental variables and descriptive species biomass (Figure 3). The eigen values for axis 1 and 2 were 0.537 and 0.463 . The Pearson correlations between species-environment $(0.960$ and 0.914) showed significant relationship between environmental variables and species distribution for the sampled points. The Monte Carlo test used to assess the significance of the first canonical axes showed that the ordination of axes 1 and 2 was statistically significant $(\mathrm{p}<0.05)$ and indicated that ordination did not occur at random.

The "intra-set" correlations showed that the DO (-0.768) and PT (-0.701) were the most important variables in the axis 1 , whereas in relation to the second axis, TDS $(-0.724)$ was the most important variable.

On the positive side of axis 1 , the samples collected during the summer were associated 
TABLE II

Relative biomass of descriptive species in sampling points $(\mathrm{P} 1=$ most preserved; $\mathbf{P} 4=$ less preserved), in winter and summer.

\begin{tabular}{|c|c|c|c|c|c|c|c|c|c|}
\hline \multirow{2}{*}{$\begin{array}{c}\text { Season } \\
\text { Class }\end{array}$} & \multirow[b]{2}{*}{ Descriptive Species } & \multicolumn{4}{|c|}{ Winter } & \multicolumn{4}{|c|}{ Summer } \\
\hline & & $\mathrm{P} 1$ & $\mathrm{P} 2$ & P3 & $\mathrm{P} 4$ & $\mathrm{P} 1$ & $\mathrm{P} 2$ & P3 & $\mathrm{P} 4$ \\
\hline \multirow{16}{*}{ Bacillariophyceae } & Amphipleura lindheimeri Grun. & & & & & 7.4 & 8.5 & 1.7 & 8.1 \\
\hline & Aulacoseira granulata (Ehr.) Simon. & & 15.7 & & & 2.0 & 5.4 & & \\
\hline & Cocconeis placentula var. acuta F. Meister & 11.2 & 4.0 & 3.9 & 6.2 & 16.1 & 16.4 & 11.3 & 12.6 \\
\hline & Cocconeis placentula var. lineata (Ehr.) van Heurck & 1.2 & 0.8 & 2.7 & 1.0 & & & & \\
\hline & Cyclotella sp. & & & & & 2.7 & & & \\
\hline & Encyonema minutum (Hilse) D.G. Mann & & 3.0 & & & & & 1.4 & 2.8 \\
\hline & Encyonema sp. & & & 2.2 & & & & & \\
\hline & Gomphonema parvulum Kütz. & 6.2 & & 6.6 & & 1.9 & 1.9 & 3.7 & 7.4 \\
\hline & Hydrosera whampoensis (Schw.) Deby & & & & & & & & 7.6 \\
\hline & Melosira varians Ag. & 9.8 & & 9.3 & 16.7 & 7.7 & & & \\
\hline & Nitzschia amphibia Grün. & 2.6 & & & & & & & \\
\hline & Pinnularia $\mathrm{cf}$. divergens W. Sm. & & & 5.8 & & & & 0.6 & \\
\hline & Pinnularia cf.subgibba Kram. & & & & 3.2 & & & & \\
\hline & Pinnularia sp. & & 2.6 & 7.9 & & & & & \\
\hline & Surirella splendida (Ehr.) Kütz. & & & & 5.8 & & 3.4 & & 1.9 \\
\hline & Ulnaria ulna (Nitzs.) Comp. & 45.5 & 3.9 & 31.6 & 15.2 & 1.9 & 12.6 & 1.6 & 8.9 \\
\hline Chlorophyceae & Coenocystis sp. & & & & & 1.7 & & & \\
\hline Chrysophyceae & Crysococcus sp. & 0.5 & 1.3 & & 0.4 & & & & \\
\hline Dinophyceae & Peridinium sp. & & & & & & & & 5.6 \\
\hline \multirow{4}{*}{ Euglenophyceae } & Euglena sp. & & & & 9.2 & & & & \\
\hline & Phacus longicauda (Ehr.) Duj. & & & & & & & 1.3 & \\
\hline & Phacus pleuronectes (O. F. Müller) Duj. & & & & & & 4.0 & & \\
\hline & Trachelomonas volvocina (Ehr.) Ehr. & 0.4 & & & 1.3 & & & & \\
\hline \multirow{2}{*}{ Zygnemaphyceae } & Closterium moniliferum (Ehr.) Ralfs & & & & & 15.5 & 13.6 & 6.2 & \\
\hline & Cumulative Biomass (\%) & 77.1 & 75.5 & 69.4 & 58.5 & 64.4 & 65.0 & 82.0 & 54.3 \\
\hline
\end{tabular}

with the highest concentrations of $\mathrm{N}^{-\mathrm{NO}_{2}}{ }^{-}$and temperature. The species grouped on this side of the axis included: Cocconeis placentula var. acuta $(\mathrm{r}=0.812)$, Closterium moniliferum (0.473), Gomphonemaparvulum(0.437), Surirellasplendida (Ehr.) Kütz. (0.224), Aulacoseira granulata (Ehr.) Simon. (0.176), Encyonema minutum (Hilse) D. G. Mann (0.145) and Peridinium sp. (0.089). On the negative side of axis 1 , the winter sampling points related with the higher DO, TP and SRP were associated. On this side of the axis, the species Trachelomonas volvocina (Ehr.) Ehr. (0.621), Chrysococcus sp. (0.581), Cocconeis placentula var. lineata (Ehr.) van Heurck (0.465), Pinnularia sp. 1(0.320), Pinnularia cf. subgibba Kram. (0.320),
Melosira varians Ag. (0.226), Nitzschia amphibia Grün. (-0.208), Euglena sp. (-0.161), Pinnularia cf. divergens W. Sm. (-0.061) and Ulnaria ulna (0.016) were grouped. Regarding axis 2 ordination, sampling units were arranged to the positive side of the axis, related to the higher concentrations of SRP (0.459). On the negative side of this axis, sampling units were disposed related to the higher values of TDS, $\mathrm{N}_{-} \mathrm{NO}_{3}{ }^{-}$and $\mathrm{N}-\mathrm{NO}_{2}{ }^{-}$, associated with the species of Amphipleura lindheimeri (-0.461), Surirella splendida (-0.476), Phacus pleuronectes (O. F. Müller) Duj. (-0.254) and P. longicauda (Ehr.) Duj. (-0.226), among others.

Regarding the preservation of the riparian vegetation, no gradient was verified in the CCA biplot. 


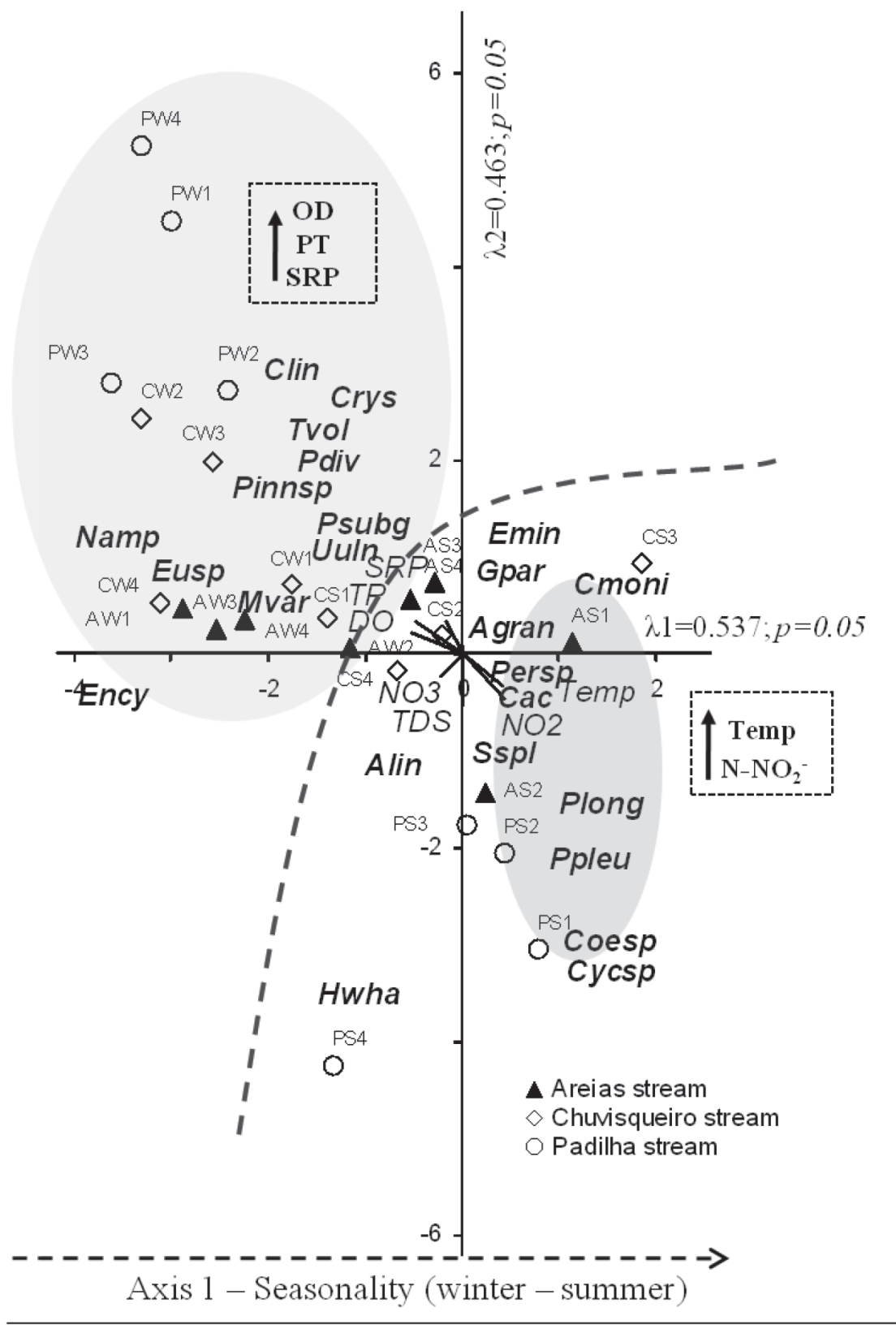

Figure 3 - Ordination by CCA (axis 1 and 2) of the sampled areas in terms of environmental and biological variables (species - biomass $\left(\mathrm{mm}^{3} . \mathrm{L}^{-1}\right) . \mathrm{A}=$ Areias stream, $\mathrm{C}=$ Chuvisqueiro stream, $\mathrm{P}=$ Padilha stream, $\mathrm{W}=$ winter, $\mathrm{S}=$ summer; 1, 2, 3, 4 = sampled areas. Alin= Amphipleura lindhiemeri; Agran = Aulacoseira granulata $;$ Cmoni $=$ Closterium moniliferum $; \mathrm{Cac}=$ Cocconeis placentula var. acuta; Clin = Cocconeis placentula var. lineata; Coesp = Coenocytis sp.; Crys = Crysococcus sp.; Cycsp = Cyclotella $\mathrm{sp} . ;$ Emin = Encyonema minutum $;$ Ency = Encyonema sp. 1; Eusp. = Euglena sp.; Gpar = Gomphonema parvulum; Hwha = Hydrosera whampoensis; Mvar = Melosira varians $;$ Namp = Nitzschia amphibia $;$ Persp = Peridinium $\mathrm{sp}$; Plong $=$ Phacus longicauda $;$ Ppleu= Phacus pleuronectes; Pdiv = Pinnularia $\mathrm{cf}$. divergens; Psub = Pinnularia $\mathrm{cf}$. subgibba $;$ Pinnsp = Pinnularia $\mathrm{sp} .1 ; \mathrm{Sspl}=$ Surirella splendida $; \mathrm{Tvol}=$ Trachelomonas volvocina; Uuln= Ulnaria ulna . 


\section{DISCUSSION}

The results showed increasing trends in the amounts of biomass, richness and diversity of potamoplankton species, from the most preserved points to the less preserved points of riparian vegetation. The integrated analysis of environmental variables and descriptive species in the studied points indicated that the gradient of riparian forest preservation was not a structuring factor of the potamoplankton community. The data showed that seasonality (winter and summer) was the main determinant of biomass patterns and presence or absence of species.

Many studies in stream ecosystems have showed seasonality as the main variable correlated with higher values of algal biomass, chlorophyll- $a$ and species richness (Rodrigues et al. 2007, Borges and Train 2009, Rossetti et al. 2009, Tavernini et al. 2011). In the present study, the higher rainfall recorded during the summer might be the explanation for the higher biomass and diversity observed in the studied streams. Apparently, the higher precipitation did not influence the water speed and, instead, might have contributed to the input of nutrient, especially nitrogen, benefiting algae development. Furthermore, the higher temperature at this station may have influenced the development of potamoplankton. Other conditions for the development of phytoplankton in the summer have been reported, such as increased temperature and luminosity (Salmaso and Zignin 2010). In general, the low values of algal biomass recorded in the studied streams are in agreement with the mean values observed in other stream ecosystems (Soares et al. 2007, Borges and Train 2009, Kireta et al. 2012).

That riparian vegetation present the ability to retain sediments, reduce nutrient and agrochemical loads, is well documented in the literature (eg, Lowrance et al. 1997, Storey and Cowley 1997, Sabater et al. 2003, Naiman et al. 2005, Borin et al. 2004, Gorsevski et al. 2008), it was expected that variations in the concentrations of physicalchemical parameters along the preservation riparian gradient would be clearly observed. Opposite to that, few variables showed clear patterns, among which stands out the highest concentration of total dissolved solids, temperature and electrical conductivity in points with less vegetation preserved. Similar condition was obtained by other authors (Johnson et al. 1997, Carpenter and Waite 2000, Vázquez et al. 2011). Regardless of this fact, the highest values of total nitrogen and total phosphorus loads contrary to the riparian vegetation gradient showed the significant impact of the vegetation absence on water quality. According to Reynolds and Davies (2001), the removal of riparian forests and their replacement by agricultural areas may increase the export of $\mathrm{P}$ up to $100 \mathrm{~kg} \mathrm{PT} \mathrm{km-}{ }^{2}$ year- ${ }^{1}$ for water bodies.

Considering the responses of potamoplankton community in the studied areas, the three major taxonomic classes identified (Bacillariophyceae, Chlorophyceae and Cyanobacteria) followed the results found in other temperate and tropical stream ecosystems (Padisák et al. 1991, O'Farrel et al. 2002, Soares et al. 2007, Rodrigues et al. 2009, Tavernini et al. 2011, Wu et al. 2012). According to Descy et al. (2012), most taxons of Bacillariophyceae are not truly planktonic, but benthic taxons in suspension, as observed in the present study (Table II) Although found in the plankton in the studied streams, Cocconeis placentula var. acuta, Amphipleura lindheimeri, Gomphonema parvulum, Melosira varians, Encyonema and Ulnaria ulna are examples of species commonly found in the benthos. The most representative of these taxons in terms of biomass may be related to the presence of riparian vegetation and the high rainfall in the summer. Rainfall removes the benthic forms from sediment to the water more easily.

Although biomass, richness and diversity patterns of potamoplankton have not demonstrated 
clear answers regarding the gradient of riparian preservation in the present study, some descriptive species identified are commonly related with nutritionally enriched environments. This is the case of Nitzschia amphibia, Melosira varians and Ulnaria ulna that are usually cited as species tolerant to eutrophication (Carpenter and Waite 2000). Similarly, Gomphonema parvulum already had its best development associated with nutrient-rich waters, especially in waters that contain domestic or agricultural wastewater (Patrick and Reimer 1975). In Brazil, this species was identified in the Pomba River (Paraná) (Soares et al. 2007) being associated with high levels of flow, temperature and alkalinity found in the rainy season. According to the classification performed by the Software Omnidia (The Diatoms Software) (Lecointe et al. 1993), Aulacoseira granulata, Cocconeis placentula var. lineata, Nitzschia amphibia are classified as indicator species of eutrophic environments, while Surirella splendida meso-eutrophic environments and Ulnaria ulna are indifferent to environment trophic status. Closterium moniliferum is regarded as a typical specie of river (Descy et al. 2012) and has also been associated to stream ecosystems near pastures (Vázquez et al. 2011). The descriptive species of group Euglenophyceae (Euglena sp., Phacus pleuronectes e $P$. longicauda) are generally found in ecosystems with high organic pollution (Safonova and Shaulo 2009, Reynolds et al. 2002).

Data analyses of the biomass, richness and diversity proved to be consistent with the River Continuum Concept (RCC) proposed by Vannotte et al. (1980), even in a small sampling scale and partially attended patterns of vegetation and increasing width of the stream. The central proposal of the RCC is that from the headwaters to downstream, variables in a lotic system exhibit a continuous gradient of conditions, including width, depth, speed, flow, temperature and entropy gain. The theory also describes the structure and function of the aquatic communities along the river course, suggesting that the distribution of species follow the abiotic gradients, mainly due to the processing of organic matter and by different trophic standards along the longitudinal gradient. So, the greatest contribution of planktonic species occurs in areas with less influence of riparian vegetation (less preserved). In the present study, these trends were recorded by phytoplankton, biomass, richness and diversity data, although not statistically significant. It is possible that the lack of significance of these results was due to the width of the riparian vegetation that may not have been sufficiently disparate to provide significant results, between the sampled areas. In literature data, a difference of $25 \mathrm{~m}$ in the width of riparian vegetation (between the most preserved and less preserved point), would be sufficient to obtain meaningful responses for the nutrients retention (Borin et al. 2004, Mayer et al. 2005), although some reviews have recommended that vegetation protected areas must be between 7 and $100 \mathrm{~m}$, being determined based on the levels of nitrogen removal that are expected (Mayer et al. 2005).

The trends observed in this study demonstrate the importance of riparian forests in the dynamics and biological structure in lotic environments. While some scientists state that efforts aimed at maintaining or restoring the water quality by the management agencies should be directed primarily to define the width of buffer zones of riparian forest (Johnson et al. 1997), there are others that re-think strategies directed to the phytoplankton bloom control in upstream areas (Hutchins et al. 2010). Anway, the interaction of longitudinal, vertical and side vectors on streams might influence their physical structure (Poole 2002), and these interactions will certainly result in different responses from biological communities, as observed in the studied streams.

In short, better water quality has been identified in areas of preserved riparian forest, as evidenced by lower nutrient concentrations. The potamoplankton structure varied seasonally, but the biomass, species 
richness and diversity did not vary significantly along the gradient preservation of riparian forest. However, the composition of descriptive species indicated possible contamination by organic matter and high nutritional contents in the studied streams, demonstrating the influence of riparian vegetation on the maintenance and quality preservation of subtropical stream ecosystems.

\section{ACKNOWLEDGMENTS}

The authors thank PETROBRAS AMBIENTAL for funding the research; the PROSUP/CAPES for granting the master's scholarship; Environmental Engineer Taison Anderson Bortolin by producing the Watershed Map and all who assisted in field work.

\section{RESUMO}

A qualidade das águas em sistemas lóticos ou lênticos sofre grande influência das atividades que são realizadas em suas margens. O presente estudo foi desenvolvido com vistas a avaliar se áreas com diferentes graus de preservação da mata ripária (de muito preservada, com $30 \mathrm{~m}$ de largura, à degradada, com menos de $5 \mathrm{~m}$ de largura) influenciam na estrutura da comunidade potamoplanctônica. O estudo foi desenvolvido em três arroios do trecho superior da Bacia Hidrográfica do Rio dos Sinos, em quatro áreas sob a influência de diferentes larguras da mata ciliar. Amostras foram coletadas para análises bióticas e abióticas no inverno (agosto/2010) e verão (janeiro/fevereiro/2011). As análises das variáveis ambientais e bióticas indicaram que a sazonalidade é a principal determinante dos padrões de biomassa, independente da largura da vegetação riparia. A comunidade potamoplanctônica estudada respondeu às condições ambientais, embora não tenham sido observadas diferenças significativas entre as áreas com diferentes graus de preservação de vegetação riparia. Tendências ao aumento da biomassa, riqueza e diversidade de espécies, e maiores concentrações de fósforo e nitrogênio total nas áreas não preservadas podem indicar que os diferentes gradientes de preservação da mata ciliar estão relacionados com a qualidade da água em arroios subtropicais e, portanto, com a estrutura do potamoplâncton.

Palavras-chave: vegetação ripária, qualidade da água, arroios, bioindicadores.

\section{REFERENCES}

American Public Health Association - APHA. 2005. Standard Methods for the examination of water and wastewater. $21^{\text {st }}$ ed. Maryland: APHA, $1368 \mathrm{p}$

BAIN MB AND STEVEnSON NJ. 1999. Aquatic habitat assessment: common methods. Betes da Maryland: AFS books, $224 \mathrm{p}$.

BITTENCOURT-OliveIRA MC. 1993a. Ficoflórula do rio Tibagi, estado do Paraná, Brasil.: I. Desmídeas, filamentosas e gêneros Gonatozygon, Penium, Pleurotaenium e Tetmemorus (Zygnemaphyceae). Semina Ciênc Biol Saúde 14: $61-73$

Bittencourt-Oliveira MC. 1993b. Ficoflórula do rio Tibagi, estado do Paraná, Brasil.: III. Actinotaenium, Cosmarium e Staurodesmus (Zygnemaphyceae). Semina Ciênc Biol Saúde 14: 86-95.

BitTenCOURT-Oliveira MC. 2002. A comunidade fitoplanctônica do rio Tibagi: uma abordagem preliminar de sua diversidade. In: MEDRI ME ET AL. (Eds), A bacia do Rio Tibagi, Londrina: FUEL, p. 373-402.

BitTENCOURT-Oliveira MC AND CASTRO AAJ. 1993. Ficoflórula do rio Tibagi, estado do Paraná, Brasil.: III. Gênero Closterium (Zygnemaphyceae). Semina Ciênc Biol Saúde 14: 74-85.

Borges PAF, Rodrigues LC, PAgIORO TA AND Train S. 2003. Spatial variation of phytoplankton and some abiotic variables in the Pirapó River -PR (Brazil) in August 1999: a preliminary study. Acta Sci Biol Sci 25: 1-8.

Borges PAF AND TRAIN S. 2009. Phytoplankton diversity in the Upper Paraná River flood plain during two years of drought (2000 and 2001). Braz J Biol 69: 637-647.

Borin M, BIGON E, ZANIN G AND FAVA L. 2004. Performance of a narrow buffer strip in abating agricultural pollutants in the shallow subsurface water flux. Environ Pollut 131: 313-321.

CARPENTER KD AND WAITE IR. 2000. Relations of habitatspecific algal assemblages to land use and water chemistry in the Willamette Basin, Oregon. Environ Monit Assess 64: 247-257.

CONLEY DJ AND MALONE TC. 1992. Annual cycle of dissolved silicate in Chesapeake Bay: implications for the production and fate of phytoplankton biomass. Mar Ecol-Prog Ser 81: 121-128.

Cugier P, Billen G, Guillaud JF, Garnier J AND MÉNESGUEN A. 2005. Modelling the eutrophication of the Seine Bight (France) under historical, present and future riverine nutrient loading. J Hydrol 304: 381-396.

DESCY JP, LEITAO M, EVERBECQ E, SMITZ JS AND DELIÈGE JF. 2012. Phytoplankton of the River Loire, France: a biodiversity and modelling study. J Plankton Res 34: 120-135. 
Dosskey MG, Helmers MJ AND EISENHAUER DE. 2011. A design aid for sizing filter strips using buffer area ratio. J Soil Water Conserv 66: 29-39.

DudGEON D. 2008. Tropical Stream Ecology, San Diego: Elsevier, $370 \mathrm{p}$.

FEPAGRO - Fundação Estadual dE PEsquisa AgropecuÁRIA. 2010. Centro de Meteorologia Aplicada. Boletim Meteorológico do Estado do Rio Grande do Sul. Agosto/2010. Disponível em: <http://www.cnpt.embrapa. br/pesquisa/agromet/pdf/Boletim_2010_08.pdf>.Acesso em: 31 jan 2013.

FEPAGRO - FundaÇÃo Estadual DE PESQuisa AgropeCuÁrIA. 2011. Centro de Meteorologia Aplicada. Boletim Meteorológico do Estado do Rio Grande do Sul. Fevereiro/2011. Disponível em: <http:/www.cnpt. embrapa.br/pesquisa/agromet/pdf/Boletim_2011_02. pdf $>$. Acesso em: 31 jan 2013.

GABEL KW, WeHr JD AND TRUHN KM. 2012. Assessment of the effectiveness of best management practices for streams draining agricultural land scapes using diatoms and macroinvertebrates. Hydrobiologia 680: 247-264.

GorsEVSKI PV, BOLL J, GOMEZDELCAMPO E AND BROOKS ES. 2008. Dynamic riparian buffer widths from potential nonpoint source pollution areas in forested watersheds. Forest Ecol Manag 256: 664-673.

HAMMER O, HARPER DAT AND RYAN PD. 2001. PAST: Paleontological statistics software package for education and data analysis. Palaeontologia Electronica 4: 1-9.

Hillebrand H, DÜrselen C-D, Kirschtel D, Pollingher U AND ZOHARY T. 1999. Biovolume calculation for pelagic and benthic microalgae. J Phycol 35: 408-424.

Hutchins MG, JOHnson AC, DeFlandre-Vlandas A, COMBER S, Posen P AND BoORMAN D. 2010. Which offers more scope to suppress river phytoplankton blooms: Reducing nutrient pollution or riparian shading? Sci Total Environ 408: 5065-5077.

Kireta AR, REAVIE ED, SGro GV, ANGRAdi TR, Bolgrien DW, HILl BH, JiCHA TM. 2012. Planktonic and periphytic diatoms as indicators of stress on great rivers of the United States: Testing water quality and disturbance models. Ecol Indic 13: 222-231.

Johnson LB, Richards C, Host GE, ARThUR JW. 1997. Landscape influences on water chemistry in Midwestern stream ecosystems. Freshwater Biol 37: 193-208.

Lecointe C, Coste M And Prygiel J. 1993. "Omnidia": software for taxonomy, calculation of diatom index and inventories management. Hydrobiologia 260/770: 509-513.

LI H, LEE JW, CAI M. 2003. Nutrient load estimation methods for rivers. International J Sediment Res 18: 346-351.

LOWRANCE R ET AL. 1997. Water quality functions of riparian forest buffer systems in Chesapeake Bay watersheds. Environ Manage 21: 687-712.

Lund JWH, KIPLING C AND LECREN ED. 1958. The inverted microscope method of estimating algal number and statistical basis of estimating by counting. Hydrobiologia 11: $143-170$.
MAYER PM, REYNOLDS JR SK, MCCUTCHEN MD AND CANFIELD TJ. 2005. U.S. Environmental Protection Riparian Buffer Width, Vegetative Cover, and Nitrogen Removal Effectiveness: A Review of Current Science and Regulations. EPA/600/R-05/118. Cincinnati, OH: U.S. Environmental Protection Agency EPA, 40 p.

MCCune B AND MefFord MJ. 2006. PC-ORD. Multivariate analysis of ecological data version 5.15. Oregon: MjM Software.

MENDES-CÂMARA FM, Moura AN AND BitTEnCOURTOLIVEIRA MC. 2002. Ficoflórula planctônica do Rio Parnaíba, Estado do Piauí - Brasil. Rev Nordestina 16: 3-21.

MÜLler-Melchers FC AND FerRANDO H. 1956. Téccnicas para El Estudio de lãs Diatomeas. Bol Inst Oceanogr 6: 93-138.

NAIMAN RJ, DÉCAMPS H AND MCClAINME. 2005. Riparia: Ecology, Conservation, and Management of Streamside Communities, Burlington: Elsevier Academic Press, 448 p.

Newbold JD, Herbert S, SWEENEy BW, KiRy P AND Alberts SJ. 2010. Water quality functions of a 15-year-old riparian forest buffer system. J Am Water Resour As 46: 299-310.

O'FArrell I, Lombardo RJ, Pinto PT AND LoEZ C. 2002. The assessment of water quality in the Lower Luján River (Buenos Aires, Argentina): phytoplankton and algal bioassays. Environ Pollut 120: 207-218.

PADISÁK J, ÁCs É, RAJCZY M AND KISS KT. 1991. Use of algae for monitoring rivers in Hungary. In: Whitton BA, Rott E and Friedrich $\mathrm{G}$ (Eds), Use of algae for monitoring rivers. Inst. Für Botanik, Uni Innsbruck, p. 123-128.

PATRICK R AND REIMER CW. 1975. The diatoms of the United States - Exclusive of Alaska and Hawaii, Philadelphia: Academy of Natural Sciences of Philadelphia. Vol. 2. Part. $1,213 \mathrm{p}$.

Perry CD, Vellidis G, Lowrance R AND Thomas DL. 1999. Watershed-scale water quality impacts of riparian forest management. J Water Res Pl-Asce 125:117-125.

PoOLE GC. 2002. Fluvial landscape ecology: addressing uniqueness within the river discontinuum. Freshwater Biol 47: 641-660.

REYNOLDS CS AND DAVIES PS. 2001. Sources and bioavailability of phosphorus fractions in freshwaters: a British perspective. Biol Rev 76: 27-64.

REYNOLDS CS, HUSZAR V, KRUK C, NASELLI-FLORES L AND MELO S. 2002. Towards a functional classification of the freshwater phytoplankton. J Plankton Res 24: 417-428.

Rodrigues LC, TraIN S, BOVO-SCOMPARIN VM, JATI S, BORSALLI CCJ AND MARENGONI E. 2009. Interannual variability of phytoplankton in the main rivers of the Upper Paraná River floodplain, Brazil: influence of upstream reservoirs. Braz J Biol 69 (Suppl 2): 501-516.

Rodrigues SC, TORGAN L AND SCHWARZBOLd A. 2007. Composição e variação sazonal da riqueza do phytoplankton na foz de rios do delta do Jacuí, RS, Brasil. Acta Bot Bras 21: 707-721.

Ros J. 1979. Práticas de Ecologia, Barcelona: Editorial Omega, $181 \mathrm{p}$. 
RossetTI G, Viaroli P AND FERrARI I. 2009. Role of abiotic and biotic factors in structuring the metazoan plankton community in a lowland river. River Res Applic 25: 814-835.

SABAter S, Butturini A, Clement JC, Burt T, Dowrick D, Hefting M, MaîtreV, Pinay G, Postolache C, RZEPECKI M AND SABATER F. 2003. Nitrogen removal by riparian buffers along a European climatic gradient: patterns and factors of variation. Ecosystems 6: 20-30.

SAFonova TA AND Shaulo SP. 2009. Phytoplankton of the Karasuk River (West Siberia) as an Indicator of Water Quality. Contemp Probl Ecol 2: 570-575.

SALMASO N AND ZIGNIN A. 2010. At the extreme of physical gradients: phytoplankton in highly flushed, large rivers. Hydrobiologia 639: 21-36.

SHANNON CE, WeAVER W. 1963. The mathematical Theory of Communication, Illinois: University of Illinois Press, Urbana, $117 \mathrm{p}$.

Silva Ca DA, Train S And Rodrigues LC. 2001. Estrutura e dinâmica da comunidade fitoplanctônica a jusante e montante do reservatório de Corumbá, Caldas Novas, Estado de Goiás, Brasil. Acta Sci 23: 283-290.

SOARES MCS, HUZCAR VLM AND ROLAND F. 2007. Phytoplankton dynamics in two tropical rivers with different degrees of human impact (Southeast Brazil). River Res Applic 23: 698-714.
STRAHLER AN. 1957. Quantitative analysis of watershed geomorphology. Eos T Am Geophys Un 38: 913-920.

STOREY RG AND COWLEY DR. 1997. Recovery of three New Zealand rural streams as they pass through native forest remnants. Hydrobiologia 353: 63-76.

Tavernini S, Pierobon E and Viaroli P. 2011. Physical factors and dissolved reactive silica affect phytoplankton community structure and dynamics in a lowland eutrophic river (Po River, Italy). Hydrobiologia 669: 213-225.

TRAIN S AND RODRIGUES LC. 1998. Temporal fluctuations of the phytoplankton community of the Baía River, in the Upper Paraná River floodplain, Mato Grosso do Sul, Brazil. Hydrobiologia 361: 125-134.

UTERMÖHL H. 1958. ZurVervollkommung der quantitativen. Phytoplankton-Methodik. Mitteilungen der International en Vereinigung für Limnologie 9: 1-38.

VANOtTE RL, Minshall GW, CUMmins KW, SEDELl JR AND Cushing CE. 1980. The River Continuum Concept. Can J Fish Aquat Sci 37: 130-137.

VÁzQuez G, AKÉ-CAstillo JA AND FaVILA ME. 2011. Algal assemblages and their relationship with water quality in tropical Mexican streams with different land uses. Hydrobiologia 667: 173-189.

Wu N, Schmalz B AND Fohrer N. 2012. Development and testing of a phytoplankton index of biotic integrity (P-IBI) for a German lowland river. Ecol Indic 13: 158-167. 